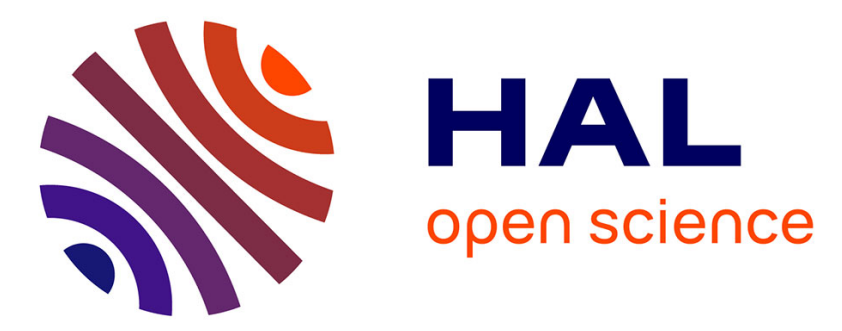

\title{
A Fully Automatic Method For Segmenting Retinal Arteries in Adaptive Optics Images
}

Nicolas Lermé, Florence Rossant, Isabelle Bloch, Michel Paques, Edouard Koch, Jonathan Benesty

\section{- To cite this version:}

Nicolas Lermé, Florence Rossant, Isabelle Bloch, Michel Paques, Edouard Koch, et al.. A Fully Automatic Method For Segmenting Retinal Arteries in Adaptive Optics Images. Pattern Recognition Letters, 2016, Special Issue on ICPR 2014 Awarded Papers, 72, pp.72-81. 10.1016/j.patrec.2015.10.011. hal-01111247

\section{HAL Id: hal-01111247 \\ https://hal.science/hal-01111247}

Submitted on 3 Feb 2015

HAL is a multi-disciplinary open access archive for the deposit and dissemination of scientific research documents, whether they are published or not. The documents may come from teaching and research institutions in France or abroad, or from public or private research centers.
L'archive ouverte pluridisciplinaire HAL, est destinée au dépôt et à la diffusion de documents scientifiques de niveau recherche, publiés ou non, émanant des établissements d'enseignement et de recherche français ou étrangers, des laboratoires publics ou privés. 


\title{
A Fully Automatic Method For Segmenting Retinal Arteries in Adaptive Optics Images
}

\author{
Nicolas Lermé ${ }^{1 *}$, Florence Rossant ${ }^{2}$, Isabelle Bloch ${ }^{3}$, Michel Paques ${ }^{4}$ \\ Edouard Koch ${ }^{5}$, Jonathan Benesty ${ }^{4}$ \\ ${ }^{1}$ MINES ParisTech, PSL Research University, Centre for Mathematical Morphology, Fontainebleau, France \\ ${ }^{2}$ Institut Supérieur d'Électronique de Paris, Issy-les-Moulineaux, France \\ ${ }^{3}$ Institut Mines-Télécom, Télécom ParisTech, CNRS LTCI, Paris, France \\ ${ }^{4}$ CIC 503, Centre Hospitalier National d'Ophtalmologie des Quinze-Vingts, Paris, France \\ ${ }^{5}$ Centre Hospitalier de Versailles, Versailles, France
}

February 3, 2015

\begin{abstract}
Adaptive optics imaging of the retina has recently proven its capability to image micrometric structures such as blood vessels, involved in common ocular diseases. In this paper, we propose an approach for automatically segmenting the walls of retinal arteries in the images acquired with this technology. The walls are modeled as four curves approximately parallel to a previously detected reference line located near the vessel center (axial reflection). These curves are first initialized using a tracking procedure and then more accurately positioned using an active contour model embedding a parallelism constraint. We consider both healthy and pathological subjects in the same framework and show that the proposed method applies in all cases. Extensive experiments are also proposed, by analyzing the robustness of the axial reflections detection, the influence of the tracking parameters as well as the performance of the tracking and the active contour model. Noticeably, the results show a good robustness for detecting axial reflections and a moderate influence of the tracking parameters. Compared to a naive initialization, the active contour model coupled with the tracking also offers faster convergence and better accuracy while keeping an overall error smaller or very near the inter-physicians error.
\end{abstract}

\section{Introduction}

This paper deals with the segmentation and the quantification of retinal blood vessels in Adaptive Optics (AO) images. This recent and non-invasive technique provides a new insight on retinal vessels and their diseases. In comparison to classical eye fundus images, AO images have a better lateral resolution [RBC $\left.{ }^{+} 11\right]$ and allow us to visualize microstructures such as photoreceptors [LWM97], capillaries [MR05, PRL ${ }^{+} 14$ ] and vascular walls [CVB12]. This technique offers a new diagnosis and prognosis investigation tool to study the diseases affecting the retinal blood vessels of small diameter $(\leq 150 \mu \mathrm{m})$ which are major causes of morbidity and mortality such as Hypertensive Retinopathy (HR) and Diabetic Retinopathy (DR). Early treatment of these diseases is crucial to avoid visual loss. This requires objective and accurate quantification of vessels features, such as wall morphometry, which can be derived from an automated segmentation of AO images. This is the topic of this paper.

Data and challenges The images used in the present study were acquired thanks to a rtx1 camera [VNL $\left.{ }^{+} 11\right]$ with flood illumination at $10 \mathrm{~Hz}$ using a $850 \mathrm{~nm}$ LED light source with a pixel resolution of $1.33 \mu \mathrm{m}$. Floodillumination systems usually produce noisy images making walls hardly visible. A common solution is to register these images and average them to increase the signal-to-noise ratio [KLBOL13]. In these images,

\footnotetext{
${ }^{*}$ Correspondig author is Nicolas Lermé (Tél: $+33(0) 1.64 .69 .48 .00$, e-mail: nicolas.lerme@mines-paristech.fr). Notice that this paper is currently under consideration at Pattern Recognition Letters.
} 


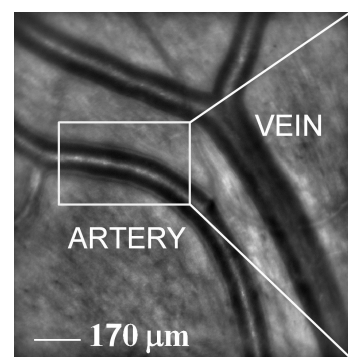

(a) $924 \times 947$ pixels

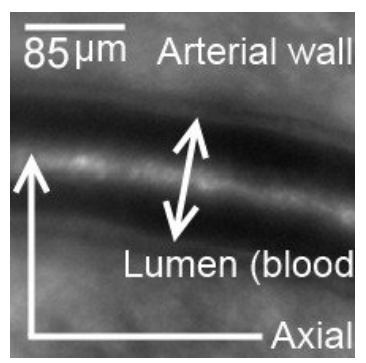

(b) $218 \times 218$ pixels

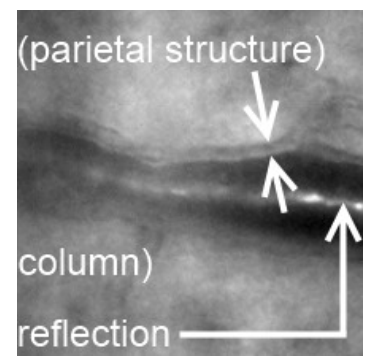

(c) $218 \times 218$ pixels

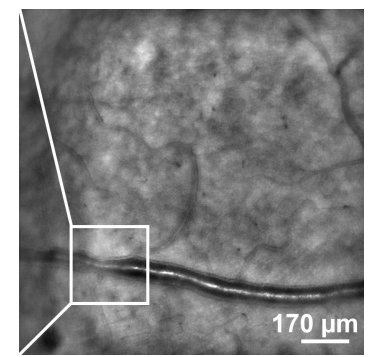

(d) $900 \times 900$ pixels

Figure 1: Examples of images acquired by the rtx1 camera and a detailed view of them for an healthy subject $(\mathrm{a}, \mathrm{b})$ and a pathological one $(\mathrm{c}, \mathrm{d})$.

blood vessels appear as dark elongated structures with a bright linear axial reflection, over a textured background. These characteristics will be exploited in the proposed method. Parietal structures (arterial walls) appear as gray lines along both sides of the lumen (blood column), with a typical thickness of about $15 \%$ of the latter $\left[\mathrm{KRB}^{+} 14\right]$ (see Figure 1).

Segmenting arterial walls in these images is however very challenging for multiple reasons: (i) the background is highly textured, (ii) the lumens are globally dark but with significant intensity variation along them, (iii) the axial reflections may locally show discontinuities or poor contrast, (iv) the outer borders of walls are low-contrasted, (v) the vessel segments can be locally blurred due to the geometry of the retina, and (vi) morphological deformations can occur in case of pathologies.

Method overview In this paper, we propose a fully automatic method for segmenting arterial walls in a selected region of interest ${ }^{1}$ in the averaged images produced by [KLBOL13]. To the best of our knowledge, this is the first method addressing this problem in such images.

To overcome the difficulties previously outlined, we propose a strategy exploiting both geometric, radiometric and topological prior information regarding vessels. More precisely, we model arterial walls as four curves approximately parallel to a common reference line located near the vessel center (axial reflection). Once this line is detected, the curves are simultaneously initialized as close as possible to the borders of walls using a tracking procedure to cope with morphological deformations along vessels (pre-segmentation). Then, these curves are more accurately positioned using a parallel active contour model where each curve evolves independently of the others towards large image gradients under a parallelism constraint [GRBP11]. This approach allows us to control the distance of the curves to their reference line, without knowing it accurately as prerequisite. This work has also permitted the physicians to establish relationships between morphometric measurements and clinical parameters $\left[\mathrm{KRB}^{+} 14\right]$.

This paper is an extension of our previous publication $\left[\mathrm{LRB}^{+} 14 \mathrm{~b}\right]$, considering still healthy subjects but with pathological ones in the same framework. Additionally, we provide more details on the method. Some steps have been improved and the method is now completely automatic, keeping in mind its use in clinical routine. The experiments and the evaluation have also been substantially expanded with the analysis of the robustness for detecting axial reflections, the influence of the tracking parameters as well as the performance of the tracking and the active contour model. This work is complementary of our previous publication $\left[\mathrm{LRB}^{+} 14 \mathrm{a}\right]$ where curves are linked to each others to improve the robustness of the model.

The rest of this paper is organized as follows. In Section 2, we detail the steps for detecting axial reflections inside vessels. Next, we introduce in Section 3 the pre-segmentation and the active contour model for segmenting arterial walls. Finally, we evaluate the performance of the method against manual segmentations in Section 4 and discuss perspectives in Section 5.

\section{Axial reflection detection}

All along this section, we consider 2D images as functions mapping pixels from $\Omega \subset Z^{2}$ into the interval $[0,1]$.

\footnotetext{
${ }^{1}$ In particular, we do not aim at segmenting the whole vascular tree.
} 


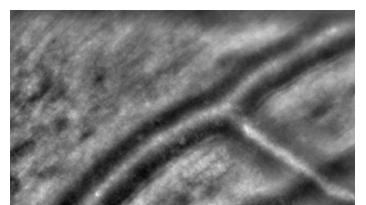

(a) Source image

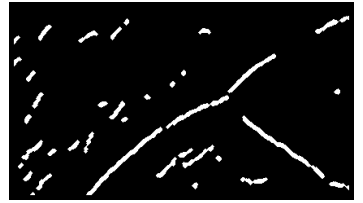

(f) $I_{E S}^{(0)}$

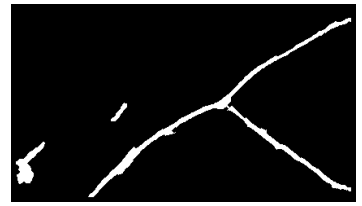

(k) $I_{E S^{\prime}}$

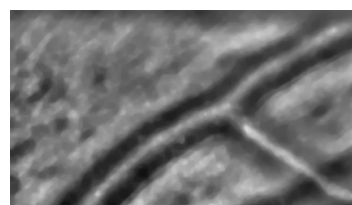

(b) $I_{P_{1}}$

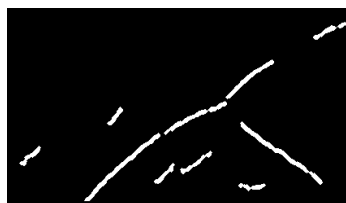

(g) $I_{E S}^{(1)}$

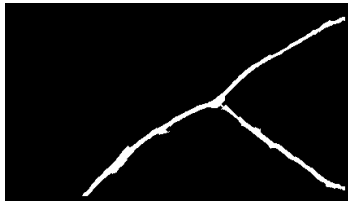

(1) $I_{E S^{\prime \prime}}$

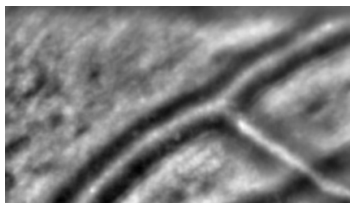

(c) $I_{P_{2}}$

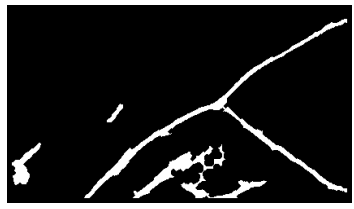

(h) $I_{E S}$

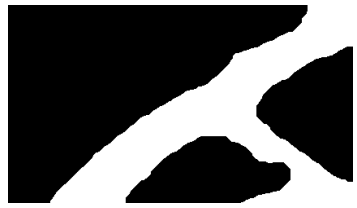

(m) $I_{L M}$

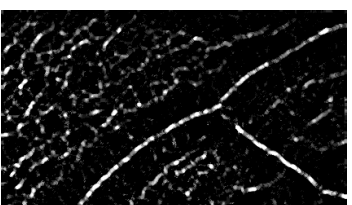

(d) $I_{T_{1}}$

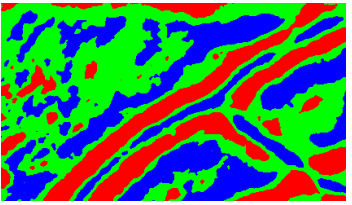

(i) $k$-mean

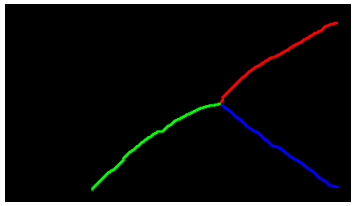

(n) Labeled axial segments

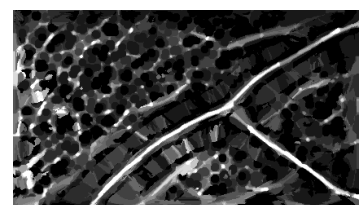

(e) $I_{T_{2}}$

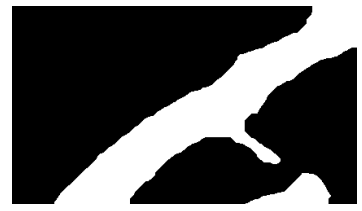

(j) $I_{D A}$

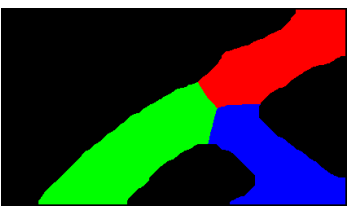

(o) Labeled lumens

Figure 2: Steps for axial reflection detection: The source image (a) is pre-processed in two different ways (b,c). Bright elongated structures are detected from (b) by top-hat (d) and adapted linear filters (e). From (d), these structures are extracted by hysteresis thresholding (f), filtered (g) and finally combined with those detected in (e) to give (h). Darkest areas are detected from (c) by k-means (i) followed by post-processing $(\mathrm{j})$. From $(\mathrm{h})$ and $(\mathrm{j})$, the most relevant axial segments are kept $(\mathrm{k}, \mathrm{l})$ and combined with $(\mathrm{j})$ to give $(\mathrm{m})$. Final axial segments (i) and lumens (j) are labeled from (d), (l) and the pruned skeleton of $(\mathrm{m})$.

\subsection{Pre-processing}

The original image (see Figure 2(a)) is first pre-processed by applying a median filter with a square structuring element of size 5 followed by a morphological closing with a circular structuring element of radius 3 , in order to enhance the axial reflection. We denote the resulting image by $I_{P_{1}}$ (see Figure 2(b)).

The source image is also denoised by a non-linear diffusion filter [WRV98] with the contrast parameter and the space regularization parameter respectively set to 0.2 and 2.0. We denote by $I_{P_{2}}$ the resulting image (see Figure 2(c)). This filter allows us smooth the vessel lumen while preserving the contrast along its edges.

\subsection{Detection of bright elongated structures}

Two filters are sequentially applied on the pre-processed image $I_{P_{1}}$ in order to further enhance the bright elongated structures. The first one is a white top-hat with a binary mask whose radius is about $1 / 3$ of the axial reflection diameter. We denote by $I_{T_{1}}$ the top-hat image (see Figure $2(\mathrm{~d})$ ). The second one is a series of adapted linear filters designed to estimate the local direction of white linear structures. The mean grey-level is calculated at every pixel $(i, j) \in \Omega$ along segments of fixed length but with different orientations, centered on it. The segment length is about 55 pixels $(\simeq 73 \mu \mathrm{m})$ and the orientation step is equal to $\Delta \theta=5^{\circ}(N=36$ filters). Let us denote by $I_{L F}^{(k)}$ the image output by the filter with orientation $k \Delta \theta, k \in\{0, \ldots, N-1\}$, and by $I_{D}$ the image storing the estimated direction:

$$
\begin{gathered}
k_{\text {opt }}(i, j)=\underset{k}{\operatorname{argmax}} I_{L F}^{(k)}(i, j), \\
I_{D}(i, j)= \begin{cases}k_{\text {opt }}(i, j) & \text { if } 0.75 I_{L F}^{\left(k_{\text {opt }}(i, j)\right)(i, j)}>I_{L F}^{\left(\left(k_{\text {opt }}(i, j)+\frac{N}{2}\right) \bmod N\right)}(i, j) \\
-1 & \text { otherwise, }\end{cases}
\end{gathered}
$$

where $x \bmod y$ is the remainder of $x$ divided by $y$. In the latter equation, the threshold 0.75 enables us to distinguish the pixels within linear bright features with well-defined local direction from all the others. From 
the top-hat image $I_{T_{1}}$, we compute a second image $I_{T_{2}}$, defined for any pixel $(i, j) \in \Omega$ as follows:

$$
I_{T_{2}}(i, j)= \begin{cases}\left(I_{T_{1}} \bullet S l_{I_{D}(i, j)}\right)(i, j) & \text { if } I_{D}(i, j) \in\{0, \ldots, N-1\} \\ I_{T_{1}}(i, j) & \text { otherwise }\end{cases}
$$

where $\bullet$ denotes the morphological closing operator and $S l_{k}$ is a binary linear structuring element whose length and orientation are respectively set to 21 pixels $(\simeq 28 \mu m)$ and $k \Delta \theta$ degrees. Compared to the image $I_{T_{1}}$, axial reflections show better properties in terms of continuity in the image $I_{T_{2}}$ (see Figure $2(\mathrm{e})$ ) but the lumens are more noisy.

Afterwards, we binarize the image $I_{T_{1}}$ using hysteresis thresholding (lower and upper thresholds are set to 0.2 and 0.6, respectively) and denote the resulting image by $I_{E S}^{(0)}$ (see Figure 2(f)). Parts of the axial reflection of vessels are thus extracted, but also other bright areas of the textured background. Further processing steps are therefore needed to discard the undesired areas. For doing so, we propose to keep the 10 largest connected components and denote the resulting binary image by $I_{E S}^{(1)}$ (see Figure $2(\mathrm{~g})$ ). To retrieve the connectivity, a geodesic reconstruction by dilation of the resulting components contained in $I_{E S}^{(1)}$ is performed in a binary mask $I_{M}$ that is dynamically built from the image $I_{T_{2}}$ as follows:

$$
I_{M}(i, j)=\mathbf{1}_{\left\{I_{T_{2}}(i, j)>S_{M}\right\}} \vee I_{E S}^{(1)}(i, j), \quad \forall(i, j) \in \Omega,
$$

with

$$
S_{M}=\left(\frac{1}{\sharp I_{E S}^{(1)}} \sum_{\substack{(i, j) \in \Omega \\ I_{E S}^{(1)}=1}} I_{T_{2}}(i, j)\right)-0.4,
$$

where $\mathbf{1}, \sharp$ and $\vee$ resp. denote the indicator function, the cardinality of a set and the logical $O R$ operator. The threshold $S_{M}$ is computed from the mean grey-level of the selected bright features in $I_{T_{2}}$, which is then lowered $(-0.4)$ in order to surely get the whole components. We denote by $I_{E S}$ the final binary image of the axial reflection components (see Figure 2(h)).

\subsection{Detection of the darkest areas}

k-means classification $(k=3)$ is performed on the pre-processed images $I_{P_{2}}$ (see Figure 2(i)) and the cluster of lowest mean intensity value provides a first binary image of the darkest regions. It is then post-processed with morphological operations to get the main connected components corresponding to the dark areas of lumens. In particular, we retain the components whose area is more than $1 / 5$ of the largest one, do a morphological closing with a circular structuring element of radius 15 and fill holes that are smaller than $10 \%$ of the area of the component having the largest surface. We denote by $I_{D A}$ the resulting image (see Figure 2(j)).

\subsection{Extraction of vascular segments by information fusion}

A first selection of vascular segments is performed based on a simple measure of the tortuosity. Let us denote by $I_{E S}^{L}$ the binary image of a tested connected component of the image $I_{E S}$. This component is retained if

$$
\frac{\sharp I_{E S}^{L}}{\sharp\left(I_{E S}^{L} \bullet S\right)}>0.8,
$$

where $S$ is a binary disk whose radius is 15 pixels. Moreover, a segment of axial reflection must lie inside a dark area, and conversely, a dark region of the lumen must contain at least one axial reflection segment. We denote by $I_{E S^{\prime}}$ the binary image made of the components satisfying (1) (see Figure 2(k)) and $I_{E S^{\prime}}^{L}$ a tested component of it. The component $I_{E S^{\prime}}^{L}$ is kept as part of an axial reflection segment if

$$
\sharp\left(I_{D A} \cap\left(I_{E S^{\prime}}^{L} \oplus S^{\prime}\right)\right)>\frac{\sharp I_{E S^{\prime}}^{L}}{5},
$$


where $\oplus$ denotes the morphological dilation operator and $S^{\prime}$ is a binary disk whose radius is 15 pixels. Notice that the radius of $S$ and $S^{\prime}$ are determined according to the minimum size of the vessels that are studied. We denote by $I_{E S^{\prime \prime}}$ the binary image made of the components satisfying (1) and (2) (see Figure 2(l)). Morphological operations are applied to $I_{D A}$, including reconstruction by dilation with the marker $I_{E S^{\prime \prime}}$, in order to get the final lumen mask $I_{L M}$ (see Figure $2(\mathrm{~m})$ ).

\subsection{Segment labeling and reconnection of branches}

We first compute the skeleton of the image $I_{E S^{\prime \prime}}$ to get the end-points of the retained segments (see Section 2.4). These end-points are then reconnected using minimal path techniques [CK97, Set99]. These techniques aim at extracting curves of minimal length, according to a Riemannian metric computed from the image and depending on the targeted application. A minimal path $C$ connecting two end-points $p$ and $q$ is obtained by minimizing the following functional:

$$
L[C]=\int_{p}^{q} \mathcal{P}(C(s)) d s
$$

where $s$ denotes the curvilinear abscissa and $\mathcal{P}$ is a potential inducing the metric defined as

$$
\mathcal{P}(x)=w_{1}\left(1-I_{T_{M}}(x)\right)^{2}+w_{2}\left(1-I_{S M}(x)\right)^{2}+w_{3}, \quad \forall x \in \Omega,
$$

where $I_{S M}$ is the pruned skeleton of the lumen mask $I_{L M}$ filtered by a Gaussian of standard deviation $\sigma$ and $w_{1}, w_{2}, w_{3} \in \mathbb{R}_{*}^{+}$are free parameters. These parameters are empirically set to $\sigma=10, w_{1}=0.5, w_{2}=0.45$ and $w_{3}=0.05$. In the latter expression, the first term is derived from the mean image image $I_{T_{M}}$ between $I_{T_{1}}$ and $I_{T_{2}}$ (considering that the values should ideally be close to one along the axial reflection) while the second one encourages the path $C$ to pass near the middle of the lumen mask $I_{L M}$. The last term is a regularization constant. The combination of the above criteria allows for a good robustness against the variety of the encountered images.

Two end-points form a candidate pair for reconnection if they belong to the same connected component in the lumen mask $I_{L M}$ and if they do not belong to the same connected component in the image $I_{E S^{\prime \prime}}$. The candidate pairs are then processed by decreasing order of the Euclidean distance (to start with points that are close to each other) and reconnected using the above procedure. A new skeleton is then calculated, providing the axial reflection of the vessels, and the vessel branches are then labeled (see Figure 2(n)). The vessel branches are individually regularized using a classical parametric active contour [KWT88] with Gradient Vector Flow [XP98]. The lumen mask $I_{L M}$ is also labeled such that every non-null pixel receives the label of the closest branch (see Figure 2(o)).

Although the above described steps rely on a number of parameters, we empirically found that they are all stable for the images studied (including those presented in Section 4).

\section{Segmentation of arterial walls}

In what follows, we detail the procedure for segmenting arterial walls of a single regularized vessel branch obtained at the end of the axial reflection detection step (see Section 2). We denote this regularized branch as the reference line $V(s)=(x(s), y(s))^{T} \in \mathbb{R}^{2}$ of the vessel, parameterized by $s$. Once obtained, this line is considered to be fixed and will therefore no longer evolve in the subsequent steps. Additionally, we choose to model the artery wall by four curves $\left\{V_{k}\right\}_{k=1}^{4}$ approximately parallel to the reference line $V$ where $V_{1}, V_{2}$ and $V_{3}, V_{4}$ represent the inner and the outer borders of this wall, respectively. These curves are defined as follows:

$$
\left\{\begin{array}{l}
V_{1}(s)=V(s)+b_{1}(s) \vec{n}(s) \\
V_{2}(s)=V(s)-b_{2}(s) \vec{n}(s) \\
V_{3}(s)=V(s)+b_{3}(s) \vec{n}(s) \\
V_{4}(s)=V(s)-b_{4}(s) \vec{n}(s)
\end{array}\right.
$$

where $\vec{n}(s) \in \mathbb{R}^{2}$ is the normal vector to the curve $V$ and $b_{k}(s) \in \mathbb{R}^{+}$is the local distance (or half-diameter) of any curve $V_{k}$ to the reference line $V$ (see Figure 3). This model establishes a direct correspondence between the points of any curve $V_{k}$ and those of the reference line $V$. The segmentation aims at computing 


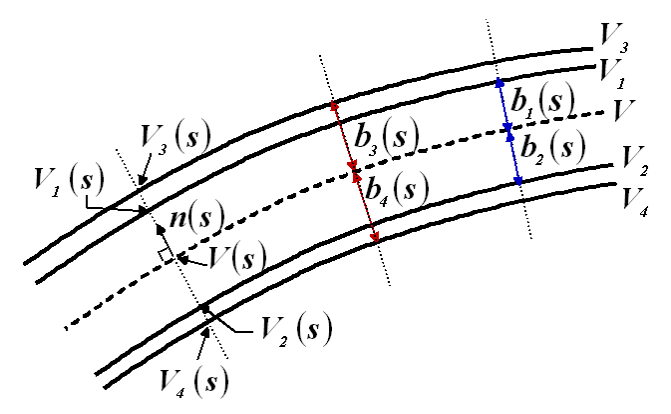

Figure 3: Parametric representation of the proposed model.

the half-diameters $\left\{b_{k}\right\}_{k=1}^{4}$ that are the most suitable for the delineation of the artery wall. By making strong (but realistic) assumptions, we first detail how these curves can be simultaneously initialized using a tracking procedure to cope with morphological deformations. Next, we relax these assumptions and present the parallel active contour model used to position these curves closer to artery walls.

\subsection{Pre-segmentation}

For convenience, we first sample the reference line $V$ into equally spaced points and denote by $V_{i}=(x(s=$ $i h), y(s=i h))^{T} \in \mathbb{R}^{2}$ the coordinates of the $i^{t h}$ point along the reference line $V$, and by $\vec{n}_{i} \in \mathbb{R}^{2}$ the associated normal vector $(h>0$ denotes the sampling step, set to 1 here). We also sample the curves representing the artery walls with, for every $i \in\{1, \ldots, \sharp V\}$

$$
\left\{\begin{aligned}
V_{i}^{1}\left(b_{i}^{\text {int }}\right) & =V_{i}+b_{i}^{\text {int }} \vec{n}_{i} \\
V_{i}^{2}\left(b_{i}^{\text {int }}\right) & =V_{i}-b_{i}^{\text {int }} \vec{n}_{i} \\
V_{i}^{3}\left(b_{i}^{\text {ext }}\right) & =V_{i}+b_{i}^{\text {ext }} \vec{n}_{i}, \quad \text { such that } b_{i}^{\text {int }}<b_{i}^{\text {ext }}, \\
V_{i}^{4}\left(b_{i}^{\text {ext }}\right) & =V_{i}-b_{i}^{\text {ext }} \vec{n}_{i}
\end{aligned}\right.
$$

where $b^{\text {int }}, b^{\text {ext }} \in \mathbb{R}^{+}$respectively denote the half-diameters of the inner and outer curves. Notice that the model (3) assumes that the inner (resp. outer) curves lie at the same distance from the reference line $V$ (i.e. $b_{1}=b_{2}=b^{\text {int }}$ and $\left.b_{3}=b_{4}=b^{e x t}\right)$. Additionally, we assume on both sides of $V$ that the walls thickness is constant along the vessels (i.e. $b^{e x t}-b^{\text {int }}=c s t$ ). Although these assumptions could appear to be somewhat strong, they are verified for a large number of images.

More generally, the goal is to obtain a robust initialization of the active contour algorithm, which in turn, will refine the curves positioning so as to reach a better accuracy. The pre-segmentation aims at simultaneously computing the half-diameters $b^{\text {int }}$ and $b^{\text {ext }}$. In this way, the robustness of the pre-segmentation against noise and poor contrast is substantially improved. For instance, this is typically useful when some of the borders of the artery walls are low contrasted since we can rely on those which are well contrasted.

Before presenting the pre-segmentation, we need to introduce some notations. First, we denote by $I: \Omega \subset \mathbb{Z}^{2} \rightarrow[0,1]$ a grayscale image and $D_{\vec{u}} I(p)$ the derivative of $I$ in the direction $\vec{u}$ at a pixel $p \in \Omega$. For a half-diameter $b \in \mathbb{R}^{+}$, a point $V_{i}$ on the reference line $V$ and a window of size $(2 r+1)$, we also define the mean local gradient along the curves $V^{1}$ and $V^{2}$ by

$$
\bar{D}^{i n t}(b, i, r)=\frac{1}{2(2 r+1)} \sum_{j=-r}^{r}\left(D_{\vec{n}_{i+j}} I\left(V_{i+j}^{1}(b)\right)+D_{-\vec{n}_{i+j}} I\left(V_{i+j}^{2}(b)\right)\right),
$$

and the mean local gradient along the curves $V^{3}$ and $V^{4}$ by

$$
\bar{D}^{e x t}(b, i, r)=\frac{1}{2(2 r+1)} \sum_{j=-r}^{r}\left(\left|D_{\vec{n}_{i+j}} I\left(V_{i+j}^{3}(b)\right)\right|+\left|D_{-\vec{n}_{i+j}} I\left(V_{i+j}^{4}(b)\right)\right|\right) .
$$

Using (4) and (5), the pre-segmentation is based on a criterion to be maximized, defined for every $i \in$ $\{1, \ldots, \sharp V\}$ by:

$$
G\left(b^{i}, b^{e}, i, r\right)=\bar{D}^{i n t}\left(b^{i}, i, r\right)+\bar{D}^{e x t}\left(b^{e}, i, r\right)
$$


This criterion encourages inner and outer curves to be located near large gradients in the image. Notice that (4) does not use absolute values on directional derivatives unlike (5). Increasing the window radius $r$ makes gradient measures more robust to noise but less reliable when strong deformations occur along vessels. This parameter therefore requires a trade-off. In what follows, we detail the steps necessary to estimate the half-diameters $b^{\text {int }}$ and $b^{\text {ext }}$ in (3).

Step 1 Instead of jointly estimating constant half-diameters $b^{\text {int }}{ }_{0}$ and $b^{e x t_{0}}$ as in [LRB $\left.{ }^{+} 14 \mathrm{~b}\right]$, we propose to estimate them as piecewise constant. In this way, the estimated half-diameters are less prone to morphological deformations while keeping a good robustness against intensity changes along the artery walls. The robustness of the tracking (see Step 3 below) is therefore improved, especially for pathological cases. Let us denote a vessel segment $k$ whose starting and ending indices in the reference line are $k_{\text {start }}$ and $k_{e n d}$, respectively. For each vessel segment $k$, the half-diameters are estimated using the criterion (6) as follows:

$$
\left(b^{i n t_{0}^{k}}, b^{e x t_{0}^{k}}\right)=\underset{\substack{b^{i}, b^{e} \in \mathbb{R}^{+} \\ b^{i}<b^{e}}}{\operatorname{argmax}} \frac{1}{2\left(k_{\text {end }}-k_{\text {start }}+1\right)} \sum_{i=k_{\text {start }}}^{k_{\text {end }}} G\left(b^{i}, b^{e}, i, r\right) .
$$

We then set $b_{i}^{\text {int } t_{0}}=b^{\text {int }} t_{0}^{k}$ and $b_{i}^{\text {ext }} t_{0}=b^{\text {ext }} t_{0}^{k}, \forall i \in\left\{k_{\text {start }}, \ldots, k_{\text {end }}\right\}$. To speed up this step, the intervals for $b^{i}$ and $b^{e}$ are restricted to typical values $\left[\mathrm{KRB}^{+} 14\right]$. In accordance to morphometric features, the piecewise constant estimation of the half-diameters is performed on vessels segments whose length is 50 pixels $(\simeq$ $66.5 \mu \mathrm{m})$. Such an approach can however fail to accurately segment the walls when deformations occur inside the same vessel segment. Estimating varying half-diameters is therefore essential to take into account these deformations.

Step 2 We determine the position along the curves having the largest contrast by maximizing the criterion (6), without regards to the vessel segments

$$
i^{*}=\underset{i \in\{1, \ldots, \sharp V\}}{\operatorname{argmax}} G\left(b^{i n t_{0}}, b^{e x t_{0}}, i, r\right) .
$$

Step 3 Finally, we jointly estimate varying half-diameters $b^{\text {int }}$ and $b^{\text {ext }}$ whose difference (the wall thickness) is constant along vessels. Again, this is achieved by using the criterion (6). These half-diameters are constructed iteratively under a regularity constraint from each side of the position $i^{*}$ found at Step 2 and using the estimates $b^{\text {int }}$ and $b^{\text {ext }}$ found at Step 1. Let us denote by $\bar{e}=\left(b_{i^{*}}^{\text {ext }}{ }_{0}-b_{i^{*}}^{\text {int }}\right)$ the wall thickness at the position $i^{*}$. For a fixed error $e$, the half-diameters are constructed as follows:

$$
b_{i}^{\text {int }}=\left\{\begin{array}{ll}
b_{i^{*}}^{\text {int }} & \text { if } i=i^{*} \\
\operatorname{argmax}_{b} E\left(b, b_{i-1}^{\text {int }}, i, e, r\right) & \text { if } i>i^{*}, \\
\operatorname{argmax}_{b} E\left(b, b_{i+1}^{\text {int }}, i, e, r\right) & \text { if } i<i^{*}
\end{array} \quad b_{i}^{\text {ext }}=b_{i}^{\text {int }}+\bar{e}+e,\right.
$$

with

$$
E\left(b, b^{\prime}, i, e, r\right)=\alpha G(b, b+\bar{e}+e, i, r)-(1-\alpha)\left(b-b^{\prime}\right)^{2},
$$

and where $\alpha \in[0,1]$ is a regularization parameter. The closer the parameter $\alpha$ is to zero, the more the right term of (8) penalizes large deviations of $b^{\text {int }}$, and conversely. To get a more robust estimate of the wall thickness $\bar{e}+e$, we propose to slightly vary $e$ around $\bar{e}$ and select the error $e^{*}$ achieving the maximum energy (8) summed along the vessel:

$$
e^{*}=\underset{e}{\operatorname{argmax}} \sum_{i>0} E\left(b_{i}^{i n t}, b_{i-1}^{i n t}, i, e, r\right) .
$$

In the latter equation, $b^{\text {int }}$ and $b^{\text {ext }}$ are constructed using (7) and (8). The resulting half-diameters $b^{\text {int }}{ }^{*}$ and $b^{e x t^{*}}$ are those found for $e^{*}$. It is not difficult to see that the assumptions made at the beginning of this section hold for these half-diameters. 


\subsection{Refined segmentation}

The model proposed by [GRBP11] simultaneously evolves two curves under a parallelism constraint. In what follows, we describe an extension of this model for extracting four curves $V_{1}, V_{2}, V_{3}$ and $V_{4}$ almost parallel to a reference line $V$. Since this line is fixed, the energy becomes

$$
E\left(V_{1}, \ldots, V_{4}, b_{1}, \ldots, b_{4}\right)=\sum_{k=1}^{4}\left(E_{\text {Image }}\left(V_{k}\right)+R\left(V_{k}, b_{k}\right)\right)
$$

where the term

$$
E_{\text {Image }}\left(V_{k}\right)=\int_{0}^{1} P\left(V_{k}(s)\right) d s,
$$

is designed to attract the curve $V_{k}$ towards large intensity gradients (see [KWT88]). In this context, the term $E_{\text {Image }}$ is based on the Gradient Vector Flow [XP98]. The role of the term $R$ in (9) is to control the variation of the distance $b_{k}$, thus imposing a local parallelism. [GRBP11] proposed a function of the derivative of $b_{k}$ with

$$
R\left(V_{k}, b_{k}\right)=\int_{0}^{1} Q\left(s, b_{k}^{\prime}\right) d s=\int_{0}^{1} \varphi_{k}(s)\left(b_{k}^{\prime}(s)\right)^{2} d s,
$$

where $\left\{\varphi_{k}\right\}_{k=1}^{4}$ are application-dependent parameters that locally control the strength of the parallelism of the curve $V_{k}$ with respect to the reference line $V$. More precisely, the larger these parameters are, the more strict the parallelism to the reference line $V$ is. Unlike previous active contour methods embedding a parallelism constraint, it is important to note that the distance between any curve $V_{k}$ and the reference line $V$ has not to be provided in the model. It is adjusted during the evolution process and can vary along boundaries. It is worth noting that the assumptions made for the pre-segmentation (see Section 3.1) are relaxed, i.e. the curves can now evolve independently of each others (instead of [LRB ${ }^{+} 14 \mathrm{a}$ ] where curves are linked to others). Also, we want to mention that the energy (9) does not ensure that $b_{1}(s)<b_{3}(s)$ and $b_{2}(s)<b_{4}(s), \forall s$. However, we never encountered such a behavior in our experiments (including for those presented in Section 4$)$.

Since the energy (9) does not have crossing terms involving different curves, the minimization can be independently done for each curve $V_{k}$. For any $k \in\{1, \ldots, 4\}$, the Euler-Lagrange equation expresses the minimization of (9) with respect to $b_{k}(s)$

$$
\frac{\partial P\left(V_{k}(s)\right)}{\partial b_{k}}-\frac{d}{d s} \frac{\partial Q\left(s, b_{k}^{\prime}\right)}{\partial b_{k}^{\prime}}=0
$$

and the evolution of the distance $b_{k}$ to the reference line $V$ is driven by

$$
\left\langle\vec{n},-\nabla P\left(V_{k}(s)\right)\right\rangle-2\left(\varphi_{k}(s) b_{k}^{\prime \prime}(s)+\varphi_{k}^{\prime}(s) b_{k}^{\prime}(s)\right)=0 .
$$

The latter equation is solved by discretizing it and introducing the time variable using standard numerical approximations of derivatives (central difference in space, backward difference in time). The resolution of the above equations stops when

$$
\max _{s}\left|b_{k}^{n}(s)-b_{k}^{n-1}(s)\right| \leq \varepsilon, \quad \forall k \in\{1, \ldots, 4\},
$$

where $b_{k}^{n}(s)$ is the distance of the curve $V_{k}$ to the reference line $V$ at iteration $n$ and $\varepsilon \simeq 0$ is an accuracy parameter.

\section{Experimental results and discussion}

\subsection{Axial reflections}

Twenty images from healthy and pathological subjects were selected to ensure the representativeness of the situations encountered by physicians during clinical routine, in terms of noise levels, contrast, morphological deformations and number of vessel branches. The selected images were validated by medical experts. In each 
image, the axial reflections of the most relevant arteries (i.e. whose lumen is visible and larger than $60 \mu m$ ) were then identified by a physician by first interactively input a few points along them and then applying a classical parametric active contour algorithm [KWT88] with Gradient Vector Flow [XP98].

When no bifurcations occur, the automatic detection of axial reflections (see Section 2) can result in fragmented reference lines due to intensity changes along them. To not overpenalize the automatic procedure, we propose to put in correspondence these lines with those obtained semi-interactively by the physician. Let us denote by $V^{A}$ and $V^{M}$ a sampled reference line obtained from the automatic procedure and from the physician, respectively. Then, we consider that these reference lines match with each other if $V^{A}$ overlaps $V^{M}$ by at least $50 \%$ of its length, or more formally, if

$$
\frac{L\left(\operatorname{Proj}\left(V^{A}, V^{M}\right)\right)}{L\left(V^{A}\right)}>0.5
$$

where $\operatorname{Proj}\left(V^{A}, V^{M}\right)$ is a subset of points of $V^{A}$ such that each of them has its nearest point in $V^{M}$ that lies at a distance less than or equal to 2 pixels and $L(x)$ denotes the piecewise linear approximation of the length of the line $x$, defined by

$$
L(x)=\sum_{i=2}^{\sharp x}\left\|x_{i}-x_{i-1}\right\|_{2} .
$$

Notice that the points of $\operatorname{Proj}\left(V^{A}, V^{M}\right)$ in (10) can be unequally spaced due to the distance threshold. The latter permits us to tolerate an imperfect positioning between the reference lines $V^{A}$ and $V^{M}$. Once the matching is done between these reference lines and to give an idea of the performance of the axial reflection detection, we propose to compare an automatic segmentation and a manual one by relying on measurements based on (i) the number of reference lines detected and (ii) their overlap. For the number of reference lines, we use:

- True Positives (TP): number of correctly matched reference lines between automatic and manual segmentations.

- False Positives (FP): number of reference lines in automatic segmentation that do not match a manual one.

- False Negatives (FN): number of reference lines in manual segmentation that do not match an automatic one.

Given a set of automatically detected reference lines $\left\{V^{A_{k}}\right\}_{k=1}^{n}$ that match with a manual reference line $V^{M}$ using (10), we also propose to use the relative overlap defined in percentage:

$$
R O\left(\left\{V^{A_{k}}\right\}_{k=1}^{n}, V^{M}\right)=\left(1-\frac{\left|L\left(V^{M}\right)-\sum_{k=1}^{n} L\left(V^{A_{k}}\right)\right|}{L\left(V^{M}\right)}\right) \times 100 .
$$

The performance of the automatic axial reflection detection against manual segmentations using the above measures is detailed in Table 1 and illustrated in Figure 4. In Table 1, we can observe that the automatic procedure offers an overall good robustness on the selected images with a mean $R O$ of $92 \%$ and a standard deviation of $8.8 \%$, meaning that most of the detected reference lines overlap well with the ideal ones. In particular, the automatic procedure demonstrates its capacity to properly manage single or multiple artery branches (see Subjects 8 and 10). However, we can notice that some images contain falsely detected axial reflections (see Subject 15). While undesired, these false detections remain minor in practice and can be easily removed by medical experts in our software. Also, we can notice that one axial reflection is missing (see Subject 11). All these particular cases are depicted in Figure 4. In particular, we observe that the detection of axial reflections is very challenging for the Subjects 11 and 15 due to the large amount of blur on some vessel segments. However, such vessels are not relevant for physicians since the parietal structures are not visible.

\subsection{Artery walls}

A sample of fourteen and seventeen images was extracted from distinct healthy and pathological subjects, respectively. These images were carefully selected by three physicians having several years of experience in the field of AO image interpretation to ensure the representativeness of the cases encountered during clinical 
Table 1: Performance of the axial reflection detection for twenty healthy and pathological subjects. False positives (FP), false negatives (FN), true positives (TP) and the relative overlap (\%) are provided (see (11) and (12)) for each image. The stars denote the images where vessel bifurcations occur.

\begin{tabular}{|c|c|c|c|c|}
\hline Subject & FP & FN & TP & RO per vessel branch (\%) \\
\hline 1 & 0 & 0 & 2 & $94.9,96.5$ \\
\hline 2 & 0 & 0 & 1 & 98.3 \\
\hline $3\left(^{*}\right)$ & 0 & 0 & 3 & $95.6,98.4,99.8$ \\
\hline $4\left(^{*}\right)$ & 0 & 0 & 3 & $89.7,95.2,96.2$ \\
\hline 5 & 0 & 0 & 1 & 99.6 \\
\hline 6 & 0 & 0 & 1 & 98.7 \\
\hline $7\left(^{*}\right)$ & 0 & 0 & 3 & $95.2,93.9,92.1$ \\
\hline $8\left(^{*}\right)$ & 0 & 0 & 5 & $84.3,59.8,99.1,96.2,95.0$ \\
\hline 9 & 0 & 0 & 1 & 95.9 \\
\hline $10\left(^{*}\right)$ & 0 & 0 & 3 & $95.7,90.7,98.1$ \\
\hline $11\left(^{*}\right)$ & 0 & 1 & 2 & $97.4,92.4$ \\
\hline $12\left(^{*}\right)$ & 1 & 0 & 3 & $91.1,83.7,83.9$ \\
\hline $13\left(^{*}\right)$ & 0 & 0 & 3 & $98.2,90.7,97.2$ \\
\hline 14 & 0 & 0 & 1 & 85.9 \\
\hline $15\left(^{*}\right)$ & 1 & 0 & 3 & $63.4,96.3,91.7$ \\
\hline $16\left(^{*}\right)$ & 1 & 0 & 3 & $97.4,90.2,71.0$ \\
\hline 17 & 0 & 0 & 1 & 99.6 \\
\hline 18 & 0 & 0 & 1 & 96.6 \\
\hline $19\left(^{*}\right)$ & 1 & 0 & 3 & $95.8,98.5,86.0$ \\
\hline $20\left(^{*}\right)$ & 0 & 0 & 3 & $86.9,80.6,97.2$ \\
\hline \hline Overall & 4 & 1 & 46 & $92.0 \pm 8.8$ \\
\hline
\end{tabular}
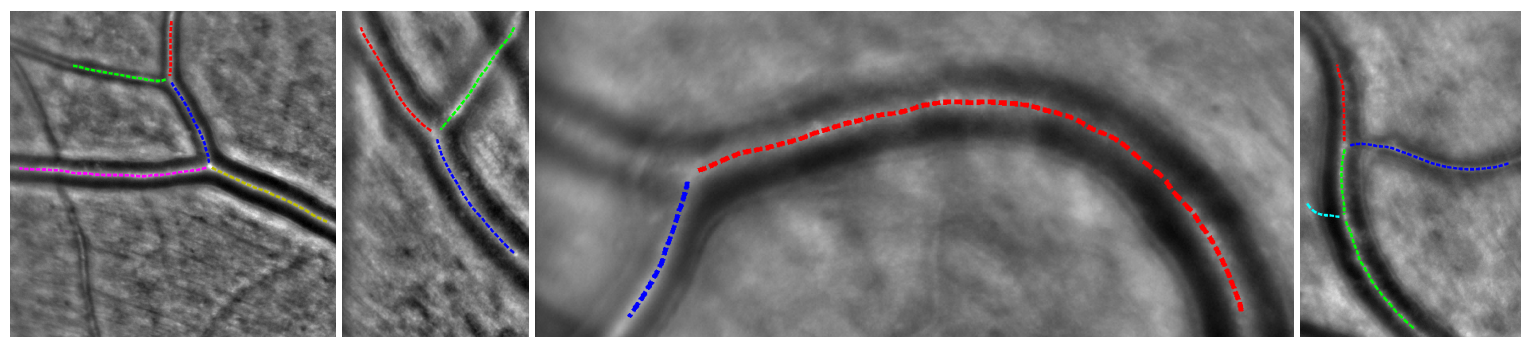

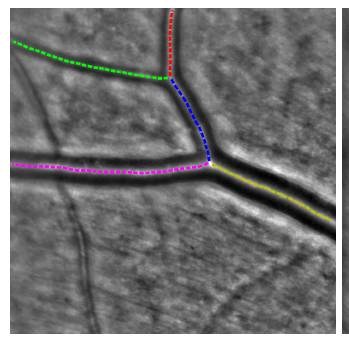

Subject 8

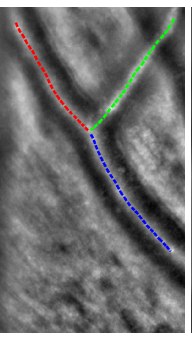

Subject 10

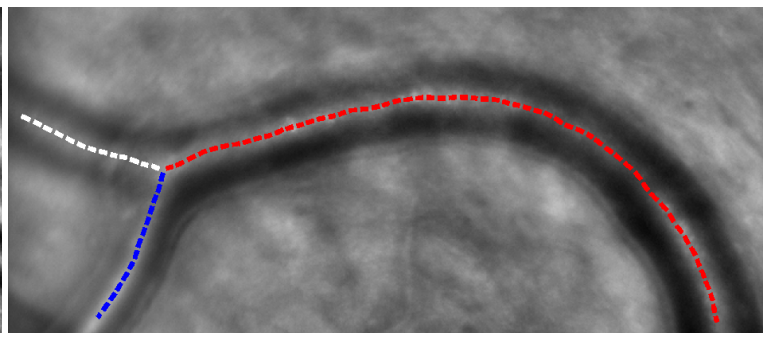

Subject 11

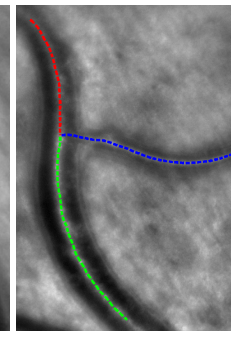

Subject 15

Figure 4: Good (half-left) and poor (half-right) results from Table 1 for the automatic detection of axial reflections for four distinct subjects. Top and bottom rows depict respectively automatic and manual segmentations, superimposed on the original image. White and cyan dashed lines correspond to missed and falsely detected reference lines, respectively. Dashed lines that are neither white or cyan correspond to correctly matched reference lines.

Table 2: Intra-physician variability for the inner diameter (ID), the outer diameter (OD) and the wall thickness (WT) (see (13), (14) and (15)). All measures are expressed in percentages and averaged over vessel and images. The numbers between parentheses correspond to a unit displacement all along a curve.

\begin{tabular}{|c|c|c|c|}
\hline & Phys $_{1} /$ Phys $_{1}$ & Phys $_{2} /$ Phys $_{2}$ & Phys $_{3} /$ Phys $_{3}$ \\
\hline ID & $3.74 \pm 3.38(1.03 \pm 0.32)$ & $4.09 \pm 3.49(1.02 \pm 0.31)$ & $3.44 \pm 2.84(1.01 \pm 0.31)$ \\
\hline OD & $2.56 \pm 2.04(0.75 \pm 0.21)$ & $3.52 \pm 2.99(0.76 \pm 0.21)$ & $3.03 \pm 2.58(0.74 \pm 0.21)$ \\
\hline WT & $20.33 \pm 20.43(6.01 \pm 2.09)$ & $27.52 \pm 31.95(6.76 \pm 3.08)$ & $22.01 \pm 22.33(6.03 \pm 2.37)$ \\
\hline
\end{tabular}


Table 3: Interest of the proposed pre-segmentation $\left(\alpha^{*}\right)$ compared to a naive one $(\alpha=0)$ in terms of convergence speed and accuracy when coupled to parallel snakes (PS). The accuracy is estimated using the absolute relative error on the inner diameter (ID), outer diameter (OD) and the walls thickness (WT) (see (13), (14) and (15)) while the convergence speed is estimated by the required number of iterations in the PS model. The inter-physicians error is also provided for comparison.

\begin{tabular}{|c|c|c|c|c|c|}
\cline { 2 - 6 } \multicolumn{1}{c|}{} & $\begin{array}{c}\text { Pre- } \\
\text { segmentation } \\
(\alpha=0)\end{array}$ & $\begin{array}{c}\text { Pre- } \\
\text { segmentation } \\
(\alpha=0)+\mathrm{PS}\end{array}$ & $\begin{array}{c}\text { Pre- } \\
\text { segmentation } \\
\left(\alpha^{*}\right)\end{array}$ & $\begin{array}{c}\text { Pre- } \\
\text { segmentation } \\
\left(\alpha^{*}\right)+\mathrm{PS}\end{array}$ & $\begin{array}{c}\text { Inter-physicians } \\
\text { error }\end{array}$ \\
\hline ID & $9.48 \pm 9.01$ & $7.78 \pm 9.94$ & $5.86 \pm 5.70$ & $5.21 \pm 5.36$ & $4.08 \pm 3.48$ \\
\hline OD & $6.34 \pm 5.02$ & $5.40 \pm 5.68$ & $3.49 \pm 3.00$ & $3.24 \pm 2.84$ & $3.31 \pm 2.80$ \\
\hline WT & $27.16 \pm 14.90$ & $30.15 \pm 28.27$ & $18.32 \pm 14.80$ & $17.59 \pm 15.75$ & $22.91 \pm 21.64$ \\
\hline Iterations & $/$ & $90.74 \pm 50.23$ & $/$ & $59.03 \pm 31.68$ & $/$ \\
\hline
\end{tabular}

Table 4: Robustness of the pre-segmentation on healthy and pathological subjects when coupled to parallel snakes (PS) by measuring the absolute relative error on the inner diameter (ID), the outer diameter (OD) and the wall thickness (WT) (see (13), (14) and (15)). The inter-physicians error is also given for comparison. The numbers between parentheses denote a unit displacement all along the curve.

\begin{tabular}{|c|c|c|c|c|}
\cline { 3 - 5 } \multicolumn{2}{c|}{} & Pre-segmentation $\left(\alpha^{*}\right)$ & Pre-segmentation $\left(\alpha^{*}\right)+$ PS & Inter-physicians error \\
\hline \multirow{3}{*}{ Training set } & ID & $5.62 \pm 7.31(1.13 \pm 0.49)$ & $5.33 \pm 7.11(1.13 \pm 0.49)$ & $4.09 \pm 3.71(1.13 \pm 0.49)$ \\
\cline { 2 - 5 } & OD & $3.46 \pm 3.45(0.83 \pm 0.31)$ & $3.35 \pm 3.51(0.83 \pm 0.31)$ & $3.24 \pm 2.79(0.83 \pm 0.31)$ \\
\cline { 2 - 5 } & WT & $16.60 \pm 12.93(6.74 \pm 2.65)$ & $16.49 \pm 13.17(6.74 \pm 2.65)$ & $21.84 \pm 17.44(6.75 \pm 2.68)$ \\
\hline \hline \multirow{3}{*}{ Test set } & ID & $5.97 \pm 4.74(0.98 \pm 0.17)$ & $5.15 \pm 4.28(0.98 \pm 0.17)$ & $4.07 \pm 3.37(0.98 \pm 0.17)$ \\
\cline { 2 - 5 } & OD & $3.51 \pm 2.77(0.71 \pm 0.11)$ & $3.19 \pm 2.46(0.71 \pm 0.11)$ & $3.34 \pm 2.81(0.71 \pm 0.11)$ \\
\cline { 2 - 5 } & WT & $19.14 \pm 15.55(5.60 \pm 1.65)$ & $18.11 \pm 16.81(5.60 \pm 1.65)$ & $23.42 \pm 23.36(5.65 \pm 1.63)$ \\
\hline
\end{tabular}

routine, i.e. in terms of noise, contrast and morphological deformations for the pathological subjects. For each image, a single artery branch was manually segmented two times by these physicians, with an interval of several weeks between the inputs to attenuate the memory effect. To measure the accuracy of a segmentation $A$ obtained with our approach against a segmentation $M$ realized by a physician, we use the absolute relative error on the inner diameter, the outer diameter and the wall thickness, resp. defined for any $i \in\{1, \ldots, \sharp V\}$ by

$$
\begin{gathered}
\delta_{i d}(M, A, i)=\frac{\left|d_{i d}(M, i)-d_{i d}(A, i)\right|}{d_{i d}(M, i)} \times 100, \\
\delta_{o d}(M, A, i)=\frac{\left|d_{o d}(M, i)-d_{o d}(A, i)\right|}{d_{o d}(M, i)} \times 100, \\
\delta_{w t_{1,2}}(M, A, i)=\frac{\left|d_{w t_{1,2}}(M, i)-d_{w t_{1,2}}(A, i)\right|}{d_{w t_{1,2}}(M, i)} \times 100,
\end{gathered}
$$

where $d_{i d}$ and $d_{o d}$ denote resp. the local inner and outer diameter while $d_{w t_{1}}$ and $d_{w t_{2}}$ denote the local wall thickness on each side of $V$. For each image, the above measures are taken where no vessel bifurcation occur and on the intersection between automatic and manual segmentations. Considering the wall thickness separately instead as a whole (as previously done in $\left[\mathrm{LRB}^{+} 14 \mathrm{~b}\right]$ ) is more sensitive but also more realistic. Notice that $\delta_{w t_{1,2}}$ is of great importance for physicians.

First, we estimate the intra-physician variability by computing the mean and the standard deviation of the measures (13), (14) and (15) for all images. To put in perspective the results and since arterial walls are very difficult to delineate up to a pixel, we also provide the mean and the standard deviation of (13) and (14) for a unit displacement along one of the four curves representing the artery walls (see Section 3.2). On each side of the reference line, (15) is for a unit displacement along one of the two curves. The resulting statistics are summarized in Table 2. As expected, the mean error on the walls thickness appears to be much larger than for the inner and outer diameter due to its high sensitivity. Also, we can also notice that the physician Phys 1 has the smallest variability on both healthy and pathological subjects for two measures out of three. Because this physician produced the most stable segmentations, we decided to choose him as a reference for the remaining of this evaluation and denote him by Phys Ref. 


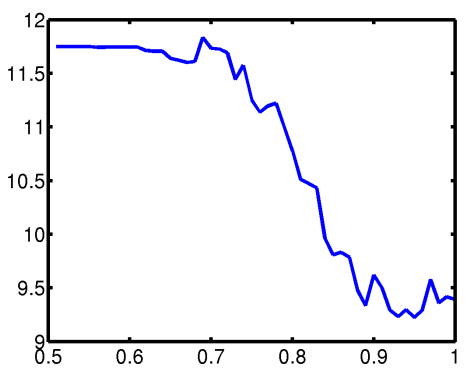

Figure 5: Sensitivity of the parameter $\alpha$ on both healthy and pathological subjects. The y-axis is the mean error of the measures (13), (14) and (15) with respect to the segmentations performed by the reference physician Phys Ref.

In what follows, the influence of the parameter $\alpha$ involved in the pre-segmentation step (see Section 3.1) is studied. In particular, the window radius $r$ is not considered here since our experiments reveal that this parameter has a limited influence on the results. We therefore choose to set $r=1$. We believe that this behavior is due to the dominant role of (8) in the tracking. To study the influence of the parameter $\alpha$, we constitute a test set and a training set using all the images from healthy and pathological subjects. The training set is composed of one third of images from healthy subjects and one third of images from pathological subjects, all randomly selected. The test set is composed of the remaining images.

Then, we search for the value of the parameter $\alpha$ giving the best accuracy against manual segmentations from the physician Phys Ref on the training set. More precisely, we choose the optimized value $\alpha^{*}$ as the one that minimizes the mean of (13), (14) and (15) along arteries and over images. We pre-segment the images from the test set using $\alpha^{*}$ and apply the parallel active contour model (PS) (see Section 3.2) on the training and test sets using the following parameter values: $\varepsilon=0.1$ and $\varphi_{k}=100, \forall k \in\{1, \ldots, 4\}$. To estimate the accuracy of our approach on the two sets of images, we again compute the mean and the standard deviation of the measures (13), (14) and (15) between the resulting segmentations and those performed by the physician Phys Ref. The inter-physicians variability is also computed for the same images and measures by comparing the segmentations performed by all the physicians, except Phys Ref, with respect to those realized by Phys Ref. All these statistics are summarized in Table 4. In this table, we can see that the resulting measures appear to be approximately in the same range, meaning good generalization properties of the parameter $\alpha$. The pre-segmentation offers a good accuracy when compared to the inter-physicians error. When parallel snakes are applied from these pre-segmentations, the accuracy is improved for all measures on both sets of images. For two measures out of three, this accuracy is for instance better for the test set compared to the inter-physicians error. A subset of the results is given in Figure 6 for two healthy subjects (first and third rows) and two pathological subjects (second and fourth rows). On the first two rows, we see that the pre-segmentation is robust to morphological deformations and is able to delineate thin walls. However, the last row depicts an extreme situation where the pre-segmentation fails due to the poor contrast on the inner borders of walls and a fake line in the textured background that mislead the tracking procedure. Also, we believe that the least performance of our approach is due to a lack of accuracy in the manual segmentations because artery walls are difficult to delineate. This issue is shown in the two last rows of Figure 6.

Figure 5 shows how the parameter $\alpha$ affects the accuracy of the pre-segmentations compared to those performed by the physician Phys Ref for both healthy and pathological subjects. Clearly, the segmentation error is not affected for $\alpha \in[0,0.65]$ and reaches a minimum for $\alpha \simeq 0.95$. Between 0.9 and 1 , the influence of the parameter $\alpha$ remains however moderate.

Finally, Table 3 demonstrates, on both healthy and pathological subjects, the interest of using a presegmentation that follows morphological deformations along vessels compared to a naive pre-segmentation that does not. For the first one, we use the optimized value $\alpha^{*}$ (see above). For the second one, we use $\alpha=0$ that leads to constant half-diameters along arteries. We then apply the PS model on all these presegmentations and compare the required number of iterations as well as the accuracy with respect to the manual segmentations from the physicians Phys Ref. In words, the pre-segmentation using $\alpha^{*}$ both leads in average to a better accuracy for all measures and diminishes the number of iterations by about $35 \%$. 


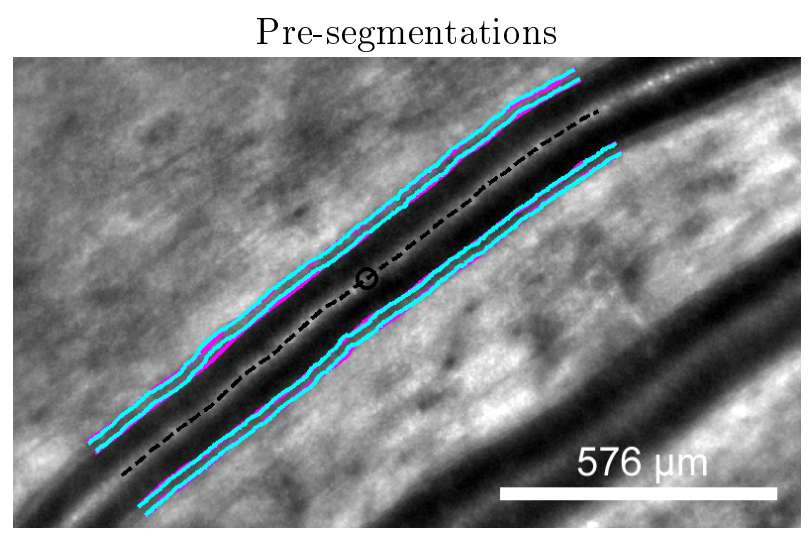

$2.85 \pm 2.14$ (ID), $1.91 \pm 1.39(\mathrm{OD}), 11.13 \pm 7.70(\mathrm{WT})$

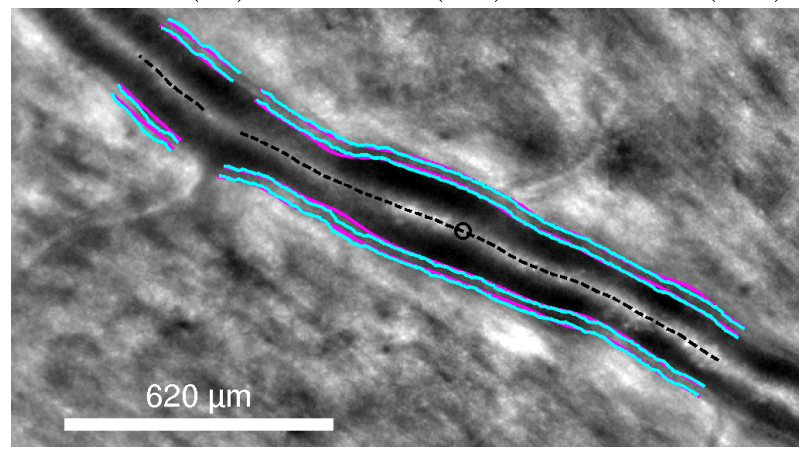

$4.30 \pm 4.54$ (ID), $2.74 \pm 2.50(\mathrm{OD}), 10.60 \pm 7.85(\mathrm{WT})$

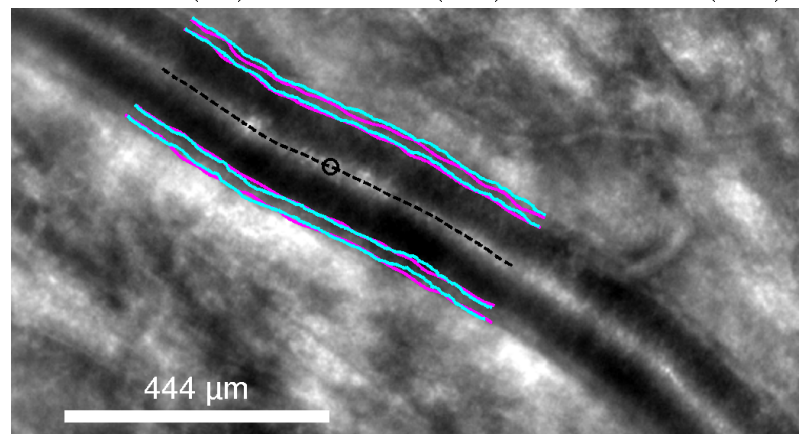

$3.48 \pm 2.42(\mathrm{ID}), 3.25 \pm 1.89(\mathrm{OD}), 21.60 \pm 20.30(\mathrm{WT})$

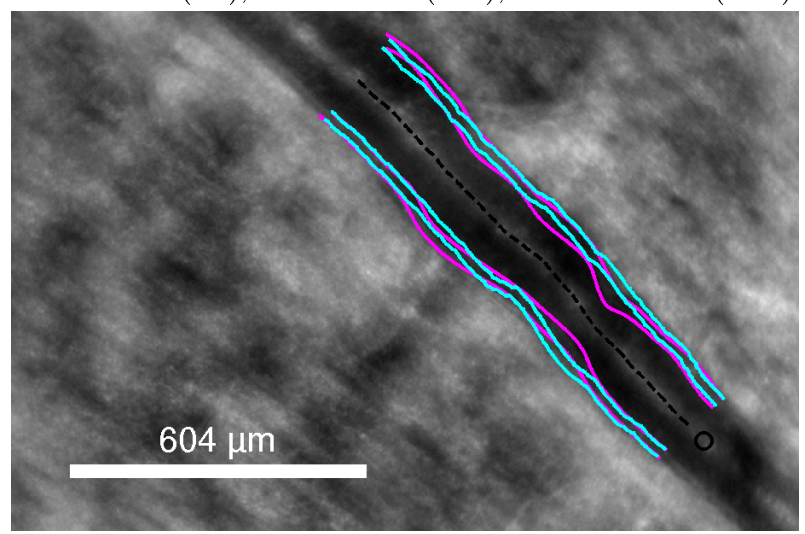

$13.71 \pm 17.00$ (ID), $7.54 \pm 6.11(\mathrm{OD}), 20.18 \pm 13.21(\mathrm{WT})$

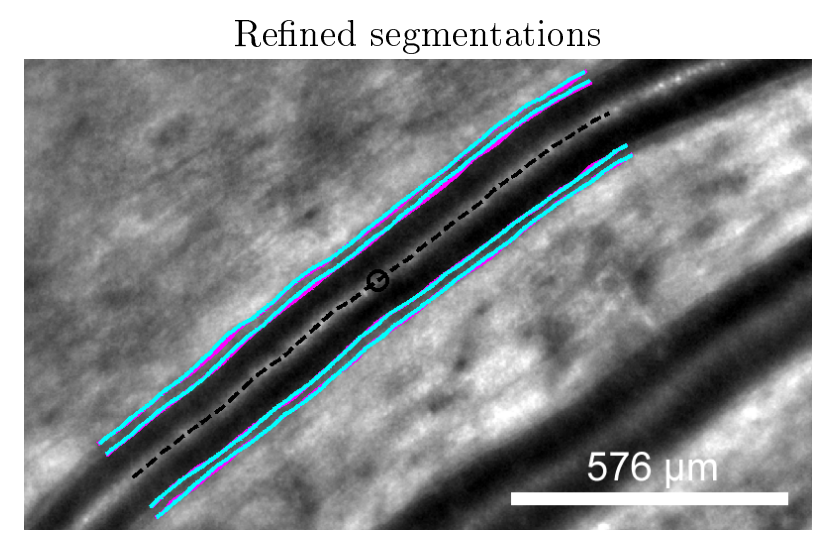

$2.76 \pm 1.56$ (ID), $1.49 \pm 1.04(\mathrm{OD}), 11.18 \pm 7.64(\mathrm{WT})$

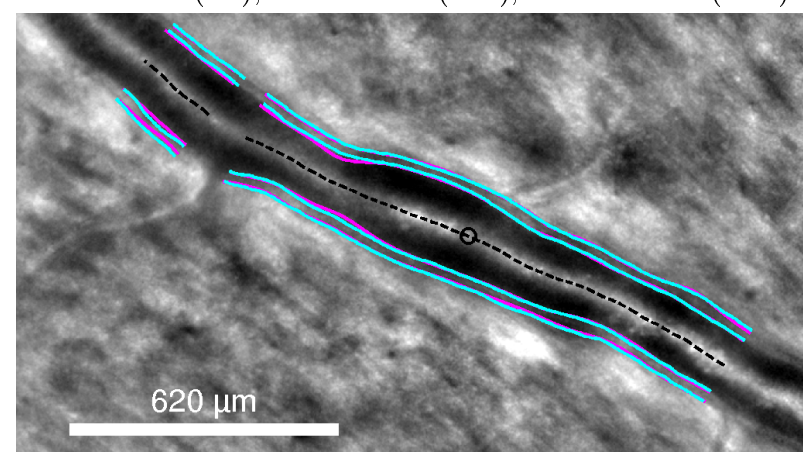

$3.56 \pm 4.14$ (ID), $2.10 \pm 1.41$ (OD), $12.26 \pm 8.80$ (WT)

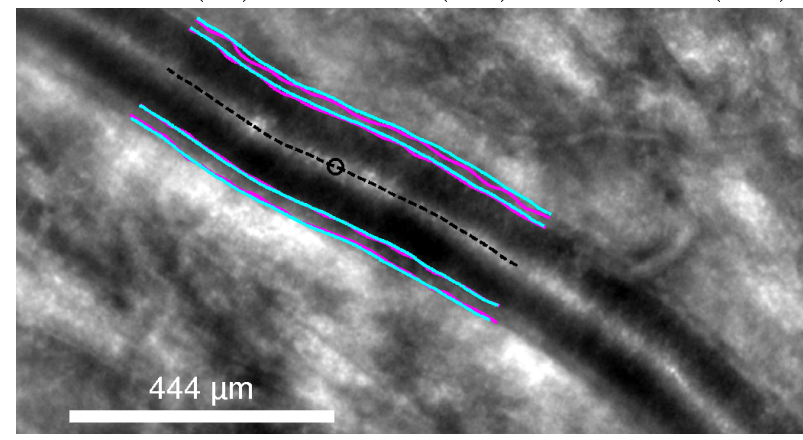

$2.17 \pm 1.65$ (ID), $4.77 \pm 1.13(\mathrm{OD}), 20.77 \pm 19.18(\mathrm{WT})$

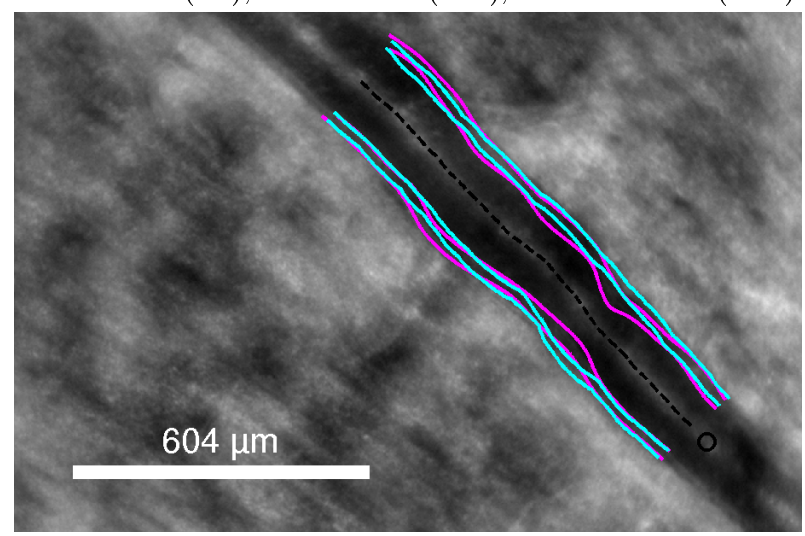

$14.03 \pm 18.13$ (ID), $7.55 \pm 6.70(\mathrm{OD}), 20.04 \pm 14.47$ (WT)

Figure 6: From top to bottom: good (upper-half) and poor (lower-half) segmentation results against the physician Phys Ref. First and third rows correspond to healthy subjects while second and fourth rows correspond to pathological ones. The left column shows pre-segmentations $\left(\alpha^{*}\right)$ while the right column shows segmentations obtained by applying parallel snakes from them. Manual and automatic segmentations are drawn in purple and cyan, respectively, superimposed on the original images. The black dashed line is the reference line $V$ while the black circle denotes the position along it from which the tracking operates in the pre-segmentation. The absolute relative error on inner diameter (ID), outer diameter (OD) and walls thickness (WT) (see (13), (14) and (15)) is indicated below each image. 


\section{Conclusion}

In this paper, we have proposed an automatic method for delineating the walls of retina arteries that apply on healthy and pathological cases. Noticeably, the results showed a good robustness for detecting axial reflections and an overall error on arterial walls measurements smaller or very near the inter-physicians error. For future work, we plan to evaluate the benefit of the coupled parallel snakes model introduced in [LRB $\left.{ }^{+} 14 \mathrm{a}\right]$ for different pathologies. Finally, notice that this work is not limited to ophtalmology applications (e.g. arterial hypertension in cardiology).

\section{Acknowledgments}

This work is funded by the ANR project ANR-12-TECS-0015-03 (2013-2015) and was performed during the post-doctoral research project of N. Lermé at the Institut Supérieur d'Électronique de Paris and Télécom ParisTech. We would like also to thank physicians for providing the manual segmentations as well as M. Lagarrigue-Charbonnier for assisting us with the algorithmic comparisons between segmentations.

\section{References}

[CK97] L.D. Cohen and R. Kimmel. Global minimum for active contour models: A minimal path approach. International Journal of Computer Vision, 24(1):57-78, 1997.

[CVB12] T.Y. Chan, D.A. Vannasdale, and S.A. Burns. The use of forward scatter to improve retinal vascular imaging with an adaptive optics scanning laser ophthalmoscope. Biomedical Optics Express, 3(10):2537-2549, 2012.

[GRBP11] I. Ghorbel, F. Rossant, I. Bloch, and M. Paques. Modeling a parallelism constraint in active contours. Application to the segmentation of eye vessels and retinal layers. In International Conference on Image Processing (ICIP), pages 445-448, 2011.

[KLBOL13] C. Kulcsár, G. Le Besnerais, E. Ödlund, and X. Levecq. Robust processing of images sequences produced by an adaptive optics retinal camera. In Optical Society of America, Adaptive Optics: Methods, Analysis and Applications, page OW3A.3, 2013.

$\left[\mathrm{KRB}^{+} 14\right] \quad$ E. Koch, D. Rosenbaum, A. Brolly, J.-A. Sahel, P. Chaumet-Riffaud, X. Girerd, F. Rossant, and M. Paques. Morphometric analysis of small arteries in the human retina using adaptive optics imaging: Relationship with blood pressure and focal vascular changes. Journal of Hypertension, 32(4):890-898, 2014.

[KWT88] M. Kass, A. Witkin, and D. Terzopoulos. Snakes: Active contour models. International Journal of Computer Vision, 1(4):321-331, 1988.

$\left[\mathrm{LRB}^{+} 14 \mathrm{a}\right]$ N. Lermé, F. Rossant, I. Bloch, M. Paques, and E. Koch. Coupled parallel snakes for segmenting healthy and pathological retinal arteries in adaptive optics images. In International Conference on Image Analysis and Recognition (ICIAR), volume LNCS 8815, pages 311-320, 2014.

[LRB $\left.{ }^{+} 14 \mathrm{~b}\right]$ N. Lermé, F. Rossant, I. Bloch, M. Paques, and E. Koch. Segmentation of retinal arteries in adaptive optics images. In International Conference on Pattern Recognition (ICPR), pages $574-578,2014$.

[LWM97] J. Liang, D.R. Williams, and D.T. Miller. Supernormal vision and high-resolution retinal imaging through adaptive optics. Journal of Optical Society America A, 14(11):2884-2892, 1997.

[MR05] J.A. Martin and A. Roorda. Direct and noninvasive assessment of parafoveal capillary leukocyte velocity. Ophthalmology, 112(12):2219-2224, 2005. 
[PRL $\left.{ }^{+} 14\right] \quad$ M. Paques, F. Rossant, N. Lermé, C. Miloudi, C. Kulcsár, J.-A. Sahel, L. Mugnier, I. Bloch, K. Loquin, and E. Koch. Adaptive optics imaging of retinal microstructures: Image processing for medical applications. In International Workshop on Computational Intelligence for Multimedia Understanding (IWCIMU), 2014.

$\left[\mathrm{RBC}^{+} 11\right]$ F. Rossant, M. Badellino, A. Chavillon, I. Bloch, and M. Paques. A morphological approach for vessel segmentation in eye fundus images, with quantitative evaluation. Journal of Medical Imaging and Health Informatics, 1(1):42-49, 2011.

[Set99] J.A. Sethian. Level Set Methods and Fast Marching Methods: Evolving Interfaces in Computational Geometry, Fluid Mechanics, Computer Vision, and Materials Science. Cambridge University Press, 1999.

$\left[\mathrm{VNL}^{+}{ }^{+11} \quad\right.$ C. Viard, K. Nakashima, B. Lamory, M. Paques, X. Levecq, and N. Château. Imaging microscopic structures in pathological retinas using a flood-illumination adaptive optics retinal camera. In Photonics West: Biomedical Optics (BiOS), volume 7885, pages 788 509+, 2011.

[WRV98] J. Weickert, B.H. Romeny, and M. Viergever. Efficient and reliable schemes for nonlinear diffusion filtering. IEEE Transactions on Image Processing, 7(3):398-410, 1998.

[XP98] C. Xu and J.L. Prince. Snakes, shapes and gradient vector flow. IEEE Transactions on Image Processing, 7(3):359-369, 1998. 